\title{
La transmisión de la "Querella de amor" del Marqués de Santillana. Estado de la cuestión, problemas y perspectivas
}

\author{
Sara RUSSO \\ Universidad Complutense de Madrid \\ sararusso@ucm.es
}

\section{RESUMEN}

En el presente artículo se considerará el decir narrativo del Marqués de Santillana "Querella de amor" en su proceso de transmisión a lo largo de los siglos XV y XVI. Se pondrá particular atención en algunos estudios que se han realizado sobre este tema para poder verificar cuál de ellos ilustra mejor la compleja situación de difusión de este poema y, a la vez, reafirmar la hipótesis de que se puede hablar de variantes de autor. Del mismo modo se mencionará la compleja problemática de los fragmentos intercalados. En última instancia, se examinará el testimonio de ML3, manuscrito hasta ahora muy poco estudiado, para poder verificar las relaciones que existen con los demás cancioneros con los que se suele vincular.

Palabras clave: Marqués de Santillana, Querella de amor, decir narrativo, transmisión poesía, ML3, variantes de autor.

\begin{abstract}
In this paper I will analyze the poem - decir narrativo - "Querella de amor" by the Marqués de Santillana and its transmission process across the XV and XVI centuries. I will focus on specific studies on this issue in order to identify which better highlights the complex situation of the diffusion of this poem while also restating the hypothesis that we can talk of variants of author. Additionally, I will draw attention to the thorny issue of the fragments inserted in the poem. Finally, I will examine the legacy of the ML3, a manuscript which has not been thoroughly studied so far, in order to determine the existing relations with the other cancioneros that are usually used as reference.
\end{abstract}

Keywords: Marqués de Santillana, Querella de amor, decir narrativo, poem transmission, ML3, variants of author.

El legado poético del Marqués de Santillana ha sido objeto de numerosos estudios y varias son las ediciones críticas de su obra. Todas ellas toman como base el llamado codex optimus, el manuscrito 2655 de la Biblioteca Universitaria de 
Salamanca (SA8) que Pedro Cátedra, entre otros, considera realizado en el mismo scriptorium del Marqués ${ }^{1}$.

Pese a lo expuesto anteriormente y en el caso específico del decir narrativo titulado "Querella de amor", objeto de mi estudio, se hace indispensable subrayar la existencia de hasta cuatro versiones del poema, representada cada una en diferentes manuscritos, con numerosas variantes que difieren del texto que nos transmite SA8 $\mathrm{y}$ de los demás manuscritos relacionados con él. Este decir narrativo tuvo una transmisión muy amplia a lo largo del siglo $\mathrm{XV}$, ya que nos ha llegado a través de un número muy elevado de manuscritos compilados o copiados durante esta época: LB2, ME1, MH1, MN54, PN8, PN12, PN13, RC1, SA7, SA8, TP1, VM1; otros tres manuscritos pertenecen al siglo XVI: YB2, ML3 y MN8, mientras que SA10 se situaría a caballo entre los dos siglos. Por último, hay que considerar que la "Querella de amor" conoció también difusión a través de la imprenta, ya que fue incluida por Hernando del Castillo en la edición de 1511 de su Cancionero General y excluida de las posteriores. En cambio, Fernández de Costantina, copiando la obra de Castillo, incluyó el poema en su Guirlanda Esmaltada cuya impresión se suele ubicar entre 1511 y 1514. Esta versión impresa dista notablemente de la que recoge el codex optimus y que hoy en día podemos leer.

El poema del que me ocupo se compone de siete coplas octosílabas entre las que se intercalan fragmentos de poemas de otros autores introducidos mediante "una formula enunciativa que subraya su carácter de cita y que constituye la solución de continuidad entre el texto y el trozo intertextual" ${ }^{\prime 2}$. En líneas generales, las citas introducidas por el Marqués en la "Querella de amor" suelen ser de cuatro versos y, siempre en líneas generales, respetan el número de sílabas de las coplas. La autoría de los fragmentos, en la mayoría de los casos, ha sido esclarecida, aunque quedan algunos cuya paternidad sigue siendo incierta y, merece la pena subrayar que, se da el caso de que dos de estos poemas citados solamente se nos han transmitido a través de este decir narrativo: "De ledo que era, triste, jay amor!, tú me tornaste,/la ora que me tiraste/la señora que me diste" y "Pois plazer non poso aver/a meu querer, de grado/serai morrer e mas non ver/meu ben perder, cuitado." Algunos testimonios presentan un apéndice final de cuatro versos, denominado finida.

El primer autor que apunta a la existencia de diferentes ramas en la trasmisión de este decir es Pérez Priego. En una nota a pie de página de su edición señala tres ramas distintas, "atendiendo al orden estrófico y a los fragmentos líricos intercalados" . En un lado sitúa la rama que considera "la más autorizada y definitiva", compuesta por SA8 y MN8. En otro lado, una segunda rama constituida por SA7, TP1, PN13, SA10, LB2, ME1, 11CG y 13*FC y, por último, una tercera

\footnotetext{
${ }^{1}$ P. Cátedra (1990), p. XXIII.

2 I. Tomassetti (2000), p. 1709.

${ }^{3}$ M. A. Pérez Priego (1983), p. 147.
} 
formada por MH1, PN12, PN8, MN54, VM1 y RC1. Los criterios en los que el editor basa esta distinción, considerando la rama de SA8 como punto de referencia, son:

-el diferente orden de las estrofas y semiestrofas, ya que en las dos últimas ramas se da una permuta de la segunda semiestrofa de la copla III con la de la copla V,

-la sustitución de algunos de los fragmentos líricos por otros: en la segunda y tercera rama, en la copla III, en lugar del intercalado lírico "Pues me fallesçió ventura..." se inserta otro, "Con tan alto poderío..." y en la tercera rama se sustituye la cita de la V copla por otra "Amor, siempre partiré",

-la presencia o no de los cuatro versos de finida: "Por ende quien me creyere/castigue en cabeça agena,/e non entre en tal cadena/do non salga, si quesiere."

Llama la atención que Pérez Priego omite mencionar dos de los cancioneros más tardíos que recogen el decir en cuestión, ML3 e YB2, de los que, sin embargo, habla en su introducción y que cita en alguna nota al texto (solo ML3). Cabe preguntarse el porqué de esta elección, ¿Los considera a ambos códices descripti o copias tardías y, por ello, sin interés? Por lo que concierne a YB2, nos informa en su introducción de que prescinde de él por considerarlo coincidente con TP1 pero, por lo que atañe a ML3, no parece expresar esa misma idea y, de todos modos, incluye en su segunda rama $13 * \mathrm{FC}$ : copia directa de $11 \mathrm{CG}$.

Pérez López, en su edición del cancionero $\mathrm{TP}^{4}{ }^{4}$, llega a establecer las filiaciones entre los diferentes manuscritos e impresos que nos transmiten la "Querella de amor" trazando un stemma del poema.

${ }^{4}$ J. L. Pérez López (1989). 


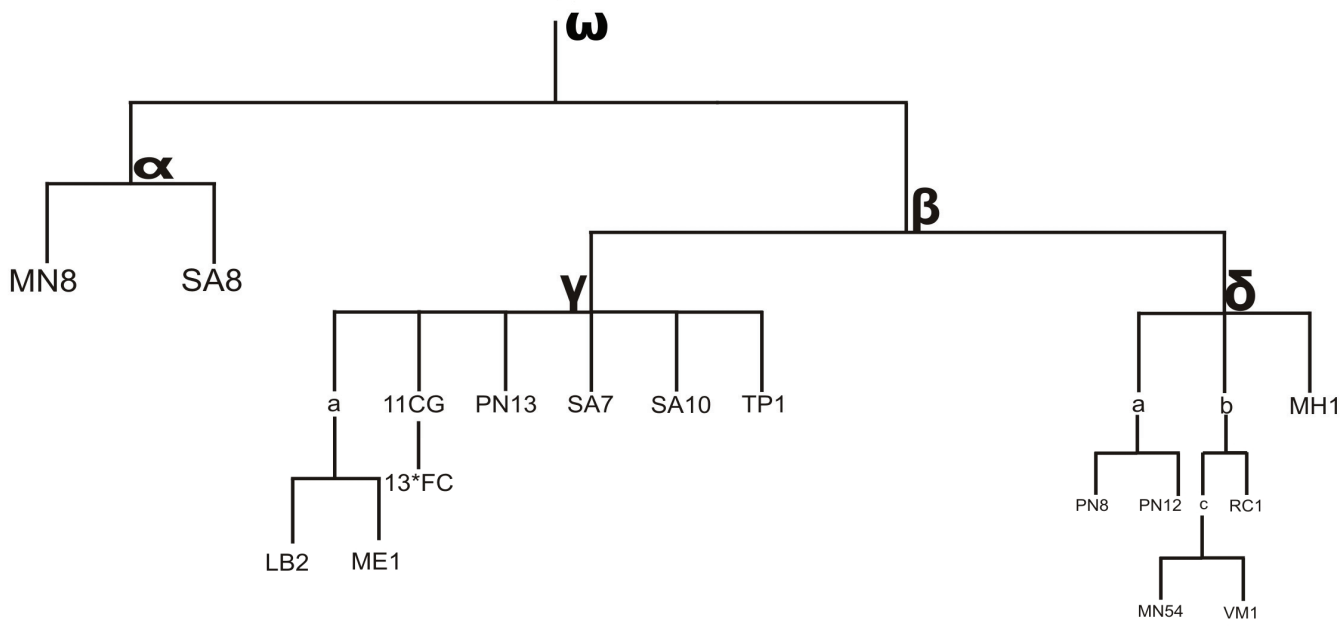

Precede al stemma un apartado en que detalla las diferencias que se dan dentro de cada rama y en el que reúne, por un lado SA7, TP1, PN13, SA10, LB2, ME1, 11CG, 13*FC y por otro MH1, PN12, PN8, MN54, VM1, RC1. Utiliza también como referencia, aunque no lo explicite abiertamente, la rama SA8. Los criterios que sigue para diferenciar las ramas son los mismos que utiliza Pérez Priego:

-el orden de las estrofas y de las semiestrofas, ya que:

- $\quad$ la rama de SA7 y la de MH1 permutan la segunda semiestrofa de la copla III con la de la copla V.

- $\quad$ solo la rama de SA7 antepone la estrofa VI a la V.

- $\quad$ solo la rama de MH1 antepone la copla VI a la IV.

-la sustitución de algunos fragmentos líricos por otros, aunque sobre este punto su explicación no sea del todo clara:

- la rama de SA7 y la de MH1 en la copla III, además del intercalado lírico "Pues me fallescio ventura...", insertan otros cuatro versos distintos que comienzan con "Con tan alto poderío...”.

- $\quad$ solo la rama de MH1 sustituye el intercalado lírico de la V por otro que comienza "Amor siempre partire".

-la presencia o no de los cuatro versos de finida.

Alude además a la edición del poema por parte de Pérez Priego, subrayando que este no emplea ML3 ni YB2. Sin explicación alguna, Pérez López no incluye estos dos códices más tardíos en su stemma, pero sí los cita e incluye en los demás stemmata que realiza de los poemas contenidos en TP1. Llega a afirmar que YB2 procede de TP1 y relaciona SA8, ML3 y MN8 al considerarlos procedentes de la 
misma familia $\alpha$. Remite a Varvaro ${ }^{5}$ por lo que concierne a la filiación de MN54, VM1 y RC1, pero no lo hace por lo que atañe a la familia de manuscritos hoy conservados en la Bibliothèque Nationale de France PN8 y PN12, de la que Varvaro se ocupa también por derivar todos, junto a los tres cancioneros procedentes de Italia (MN54, VM1 y RC1), de un antecedente común. Parece que el editor se propone hacer exclusivamente un stemma del poema pero luego, inevitablemente, se ve influenciado en su propósito por las relaciones que los estudios sobre los cancioneros manuscritos han evidenciado a lo largo del tiempo. Señala, por ejemplo, que MH1, PN12, PN8, MN54, VM1, RC1, entre otras coincidencias, mantienen (MH1, PN12) o no (PN8, MN54, VM1, RC1) la finida. Luego, en el stemma, reúne los manuscritos italianos y los franceses según las familias establecidas por Varvaro dejando apartado a MH1 que, sin embargo, junto con PN12, es el único de la rama $\delta$ que presenta la finida. Quizás, por lo que concierne la transmisión de la "Querella de amor", habría que relacionar más estrechamente MH1 y PN12 e incluir PN8 en la rama de la familia de cancioneros italianos, aunque este modus operandi entre en abierta contradicción con el análisis de la transmisión del conjunto de códices mencionados.

El último autor que nos habla de la transmisión de este poema es Brian Dutton. La subdivisión que él hace resulta ser la más cautelosa, ya que distingue cinco versiones básicas del decir, agrupándolas "según la secuencia de las citas y la presencia o ausencia del cuarteto final", stemma. Incluye todos los manuscritos, incluso los más tardíos, pero omite $13 * \mathrm{FC}$ por considerarlo copia directa de 11CG. De este modo divide:

$$
\begin{aligned}
& \text {-1. SA8, MN8, ML3 } \\
& \text {-2. SA7, TP1, PN13, SA10, 11CG, YB2 } \\
& \text {-3. LB2, ME1 } \\
& \text {-4. MH1, PN12 } \\
& \text {-5. PN8, MN54, VM1, RC1 }
\end{aligned}
$$

Los criterios que utiliza Dutton para establecer estas versiones distan ligeramente de los empleados por Pérez Priego y Pérez López, ya que el inglés parece prescindir del orden de las estrofas basándose exclusivamente en el orden de los fragmentos líricos y en la presencia o no de la finida. De todos modos, considero que, aunque no lo mencione, inevitablemente, al considerar el orden de las citas, tuvo que tomar en cuenta también el orden de las coplas. Reúne MH1 y PN12 bajo la misma versión mientras que agrupa aparte a PN8, MN54, VM1 y RC1. Todos estos testimonios presentan exactamente el mismo orden de citas y estrofas (pese a

5 A. Varvaro (1964).

${ }^{6}$ B. Dutton (1990-1991), v. 7, p. 13. 
la laguna de dos coplas y dos citas en PN12) pero, como indiqué anteriormente, MH1 y PN12 incluyen la finida y, por eso mismo, Dutton considera más correcto separarlos. Por lo que concierne a LB2 y ME1 -que los demás editores suelen considerar conjuntamente a SA7, TP1, PN13, SA10, 11CG, YB2-, Dutton los separa por presentar el cancionero londinense (LB2) y el de Módena (ME1), dos citas más extensas con respecto a la versión dos (en los siguientes ejemplos representada por SA7), hecho que rompe la estructura misma del poema, compuesto de coplas de ocho versos entre las que se intercalan citas de cuatro versos:

\begin{tabular}{|c|c|c|}
\hline $1^{\text {a }}$ CITA & Versión dos: SA7 & Versión tres: ME1 y LB2 \\
\hline & Con tan alto poderio & Con tan alto poderio \\
\hline & amor nunca fue iuntado & amor nunca fue iuntado \\
\hline & ni con tal ergullo et brio & nin con tan argullo et brio \\
\hline & qua yo vi por mi pecado & qual yo vi por mi pecado \\
\hline & & contra mi que fuy sandio \\
\hline & & de nodado en yr veer \\
\hline & & el su muncho gran poder \\
\hline & & et muy alto sennorio \\
\hline
\end{tabular}

\begin{tabular}{|l|l|l|}
\hline $\mathbf{2}^{\text {a }}$ CITA & \multicolumn{1}{|c|}{ Versión dos: SA7 } & \multicolumn{1}{c|}{ Versión tres: ME1 y LB2 } \\
\hline & Catiuo de minya tristura & Catiuo de mi tristura \\
\hline & $\begin{array}{l}\text { ya todos prenden } \\
\text { espanto }\end{array}$ & ya todos prenda el espanto \\
\hline & e preguntan que ventura & e pregunta en ventura \\
\hline & $\begin{array}{l}\text { es que matormenta } \\
\text { tanto }\end{array}$ & fue que me atormenta tanto \\
\hline & & que non se en el mundo amigo \\
\hline & & a quien mas de mi quebranto \\
\hline & & diga desto que vos digo \\
\hline
\end{tabular}

y un orden diferente de los fragmentos intercalados (cito sólo el primer verso del fragmento): 


\begin{tabular}{|l|l|l|}
\hline $\begin{array}{c}\text { Posición de la } \\
\text { cita en el texto }\end{array}$ & \multicolumn{1}{|c|}{ Versión dos: SA7 } & ME1 y LB2 \\
\hline $\begin{array}{l}1^{\mathrm{a}} \text { cita } \\
\text { introducida }\end{array}$ & Amor cruel et brioso & Amor cruel e brioso \\
\hline $\begin{array}{l}2^{\mathrm{a}} \text { cita } \\
\text { introducida }\end{array}$ & De ledo que era triste & De ledo que era triste \\
\hline $\begin{array}{l}3^{\mathrm{a}} \text { cita } \\
\text { introducida }\end{array}$ & Con tan alto poderio & Con tan alto poderio \\
\hline $\begin{array}{l}4^{\mathrm{a}} \text { cita } \\
\text { introducida }\end{array}$ & Catiuo de minya tristura & Crueldad e gran tormento \\
\hline $\begin{array}{l}5^{\mathrm{a}} \text { cita } \\
\text { introducida }\end{array}$ & Crueldat e trocamento & Catiuo de mi tristura \\
\hline $\begin{array}{l}6^{\mathrm{a}} \text { cita } \\
\text { introducida }\end{array}$ & Pero te siruo sin arte & Pero si te siruo sin arte \\
\hline $\begin{array}{l}7^{\mathrm{a}} \text { cita } \\
\text { introducida }\end{array}$ & $\begin{array}{l}\text { Pues que plazer non } \\
\text { poso hauer }\end{array}$ & Pues que plazer non puedo aver \\
\hline
\end{tabular}

Considerando cuanto he expuesto hasta ahora, se evidencia un hecho fundamental: parece que los tres autores utilizan en general los mismos criterios. Lo único que distancia a Dutton de los otros dos es el no mencionar el orden de las estrofas y semiestrofas aunque, como ya apunté anteriormente, creo inevitable que considerara el orden de las coplas aunque no lo señale. Pese al empleo de los mismos criterios, los resultados son diferentes. La subdivisión de Dutton resulta ser la más prudente, ya que su partición entre los testimonios no implica necesariamente -como es el caso del stemma de Pérez López, que hace proceder todos los testimonios de un único original común $\omega-$, que la "Querella de amor" haya tenido una única redacción. Además, Dutton parece fijarse exclusivamente en el texto del poema mientras que Pérez López, como ya señalé, no prescinde de las filiaciones históricamente establecidas entre los cancioneros. Pérez Priego, por su parte, no alude en ningún momento a la posibilidad de una única redacción del decir, pero parece también que su partición se hace eco de estudios previos sobre las relaciones entre los códices y, a la hora de tener que editar el poema, escoge SA8 por pertenecer a la rama más autorizada y definitiva, ya que probablemente fue revisada por el mismo poeta. Merece la pena subrayar que los criterios empleados por los tres autores revelan que ninguno de ellos determina su división entre los testimonios en función de los errores. Probablemente esto se deba, a pesar de que ninguno de los tres lo explicite abiertamente, a que los errores no son suficientes ni en términos cualitativos ni cuantitativos, hecho que comporta la necesidad de tomar 
en consideración exclusivamente las variantes, por lo menos por lo que atañe al establecimiento de una primera y general repartición entre los testimonios.

Hay que considerar también que dos de las ediciones modernas fundamentales de la obra del Marqués de Santillana, la de Pérez Priego ${ }^{7}$ y la de Gómez Moreno y Kerkhof ${ }^{8}$, toman como texto base SA8. El primer editor enmienda los fallos y lagunas del códex optimus con MN8 y, allí donde considere otros testimonios, lo explicita al comienzo de cada obra, mientras que los otros dos editores enmiendan los fallos y lagunas de SA8 con otros testigos sin dar especial relevancia a MN8. Es este último una copia tardía del siglo XVI de un cancionero de autor que probablemente reunió el mismo Marqués con el fin de recopilar en una única obra todos sus escritos, del que se sabe que SA8 es también copia muy cuidada y que a su vez, como ya vimos, salió del scriptorium de Íñigo López de Mendoza. El códex optimus es un manuscrito que "acaso sea la ultima versión ne varietur de un cancionero de Santillana como tal", y su fecha de compilación se suele ubicar en el año de 1455; tres años después, en 1458, el Marqués muere. La cercanía entre la fecha de compilación de SA8 y la de la muerte del poeta sugiere que los poemas en él trasmitidos corresponden principalmente a un estado maduro de la actividad poética de Santillana, siempre y cuando se considere la posibilidad de hablar de variantes de autor. En el caso de la "Querella de amor" parece inevitable considerar esta eventualidad pese a que el stemma de Pérez López nos induzca hacia el camino contrario. El mismo Pérez Priego, apoyándose en Menéndez Pidal ${ }^{10}$ y Lapesa ${ }^{11}$, afirma, en relación a todo a la obra del Marqués de Santillana que existe "[...] una nutrida serie de variantes de autor... [que] se manifiesta con sorprendente frecuencia y esplendor en los decires narrativos" ${ }^{\prime 2}$. De esta manera resulta evidente que SA8 y MN8 (e incluiría también ML3) nos transmiten una "Querella de amor" que corresponde a la última voluntad del autor mientras los demás cancioneros nos enseñan posiblemente la existencia de un primitivo e inicial estadio del poema que ha ido evolucionando, quizás bajo la supervisión del mismo autor. Así que es probable que alguna de las versiones que tenemos sea la más próxima al poema que Íñigo López de Mendoza escribió originariamente en su juventud y con anterioridad a 1437, fecha indicada por Lapesa como término ante quem ${ }^{13}$.

Acaso el orden diferente de las coplas y de los fragmentos entre los varios testimonios no es indicativo para poder hablar de variantes de autor, ya que puede deberse a un desorden entre los cuadernillos de un códice que colocaríamos en un

${ }^{7}$ M. A. Pérez Priego (2008).

${ }^{8}$ A. Gómez Moreno, M. P. A. M. Kerkhof (1988).

${ }^{9}$ P. Cátedra (1990), p. XXIII.

${ }^{10}$ R. Menéndez Pidal (1941).

${ }^{11}$ R. Lapesa (1956).

${ }_{12}$ M. A. Pérez Priego (2008), p. 34.

${ }^{13}$ Lapesa (1956), p. 86. 
eslabón intermedio entre un primera escritura del poema y los testimonios que nos han llegado. Además, como bien afirma Blecua, "en los textos en verso no resulta extraño encontrar inversiones del orden de las estrofas y de los versos"14. Pero, en la "Querella de amor" se dan ejemplos mucho más evidentes que inducen a confirmar la existencia de variantes de autor. Un ejemplo muy esclarecedor es el siguiente ${ }^{15}$ :

\begin{tabular}{|l|l|}
\hline $\begin{array}{l}\text { Versión 1, } \\
\text { MN8 }\end{array}$ & $\begin{array}{l}\text { Pues me fallesçio ventura/ en el tiempo del plazer/ non espero auer folgura/ } \\
\text { mas por siempre entristecer }\end{array}$ \\
\hline $\begin{array}{l}\text { Versión 2, } \\
\text { SA7 }\end{array}$ & $\begin{array}{l}\text { Con tan alto poderio/ amor nunca fue iuntado/ ni con tal ergullo et brio/ qua yo } \\
\text { vi por mi pecado }\end{array}$ \\
\hline $\begin{array}{l}\text { Versión 3, } \\
\text { ME1 }\end{array}$ & $\begin{array}{l}\text { Con tan alto poderio/ amor nunca fue iuntado/ nin con tan argullo et brio/ qual } \\
\text { yo vy por mi pecado/ contra mi que fuy sandio/ denodado en yr veer/ el su } \\
\text { muncho gran poder/ et muy alto sennorio }\end{array}$ \\
\hline $\begin{array}{l}\text { Versión 4, } \\
\text { MH1 }\end{array}$ & $\begin{array}{l}\text { Con tan alto poderio/ amor nunca fue iuntado/ nin con tanto orgullo et brio/ } \\
\text { commo vy yo mal fadado }\end{array}$ \\
\hline $\begin{array}{l}\text { Versión 5, } \\
\text { MN54 }\end{array}$ & $\begin{array}{l}\text { Con tan alto poderio/ amor nunca fue iuntado/ nin con tant orgullo brio/ como } \\
\text { ui por mi peccado }\end{array}$ \\
\hline
\end{tabular}

Como se puede notar ME1, junto a LB1 que en el ejemplo no cito, son los únicos testimonios que reproducen integralmente la primera estrofa de un poema de Macías mientras que SA7, MN54 y MH1, y los demás testimonios que estos tres cancioneros representan en el ejemplo, solamente reproducen los primeros cuatro versos. La versión primera, representada aquí por MN8, merece mención aparte. SA8 se caracteriza por algunas lagunas a lo largo del códice debidas a la pérdida de algunos folios, una de estas afecta a la "Querella de amor", ya que la falta de un folio provoca la pérdida de 47 versos. El ejemplo arriba mencionado recoge aquellos versos que faltan en el códex optimus. De todos modos, ya que ML3 también reproduce este fragmento, se podría suponer que antes de la pérdida, SA8 también lo reprodujera. Parece evidente que la cita "Pues me fallesçio ventura", a pesar de recogerse en la rama "más autorizada", no estaría en un posible antígrafo de la versión dos (SA7, TP1, PN13, SA10, 11CG, YB2) y de la cinco (PN8, MN54, VM1, RC1) ya que habría que suponer que, independientemente, dos ramas diferentes innovaran de la misma manera introduciendo otro fragmento: "Con tan alto poderío"; atribuir semejante innovación a un copista resulta de lo más atrevido. No queda sino considerar una intervención directa del autor que, en un momento

${ }_{15}^{14}$ A. Blecua (1983), p. 23.

15 Desde aquí en adelante seguiré refiriéndome a las cinco versiones del poema que establece Dutton utilizando, en los ejemplos, siempre el mismo testimonio como representante de cada versión. 
dado, por cuestiones estilísticas quizás, decidiera cambiar un fragmento lírico por otro.

Otro ejemplo parecido ${ }^{16}$ (es este otro caso en que los fragmentos intercalados, los que reproducen aquí la versión uno y la dos, son de tres versos en lugar de los cuatro versos de los que se constituyen todas las demás citas):

\begin{tabular}{|l|l|}
\hline $\begin{array}{l}\text { Versión 1, } \\
\text { MN8 }\end{array}$ & Pero te siruo sin arte/ ay amor amor amor/ gran cuyta de mi nunca se parte \\
\hline $\begin{array}{l}\text { Versión 2, } \\
\text { SA7 }\end{array}$ & Pero te siruo sin arte/ ay amor amor/ gran cuyta de mi nunca se parte \\
\hline $\begin{array}{l}\text { Versión 3, } \\
\text { ME1 }\end{array}$ & $\begin{array}{l}\text { Pero te siruo sin arte/ ay amor amor asi/ grande cuita de mi/ nunca jamas se } \\
\text { parte }\end{array}$ \\
\hline $\begin{array}{l}\text { Versión 4, } \\
\text { MH1 }\end{array}$ & $\begin{array}{l}\text { Amor çierto partire/ de vos sienpre me quexando/ pues por vos seruir } \\
\text { loando/ soy en tiempo que morre }\end{array}$ \\
\hline $\begin{array}{l}\text { Versión 5, } \\
\text { MN54 }\end{array}$ & $\begin{array}{l}\text { Amor siempre partire/ de uos assy me quexando/ pues por uos seruir loando/ } \\
\text { soy a tiempo de morire }\end{array}$ \\
\hline
\end{tabular}

Una vez más, unos testimonios intercambian un fragmento por otro, apuntando, con evidencia creciente, que nos encontramos frente a unas variantes de autor que imposibilitan trazar un stemma como el que nos proporciona Pérez López.

Los fragmentos que el Marqués de Santillana inserta son versos de poesías de otros autores que circulaban ya en aquel momento. Es inevitable suponer que estas poesías también tuvieran diferentes variantes, de autor o no. Este tema es extremadamente complejo y merecería ser tratado de manera mucho más profunda pero, por ahora, solo se aludirá a ello por ser imprescindible a la hora de tratar el decir narrativo objeto de estudio y, sobre todo, por ser uno de los elementos fundamentales que apoyan la hipótesis de las variantes de autor. Se hace así imprescindible un cotejo de las citas que introduce el Marqués de Santillana con los poemas originales de los que proceden. Uno de los ejemplos citados anteriormente, el fragmento "Con tan alto poderio", nos muestra que, entre los diferentes testimonios de la "Querella de amor", este se recoge en diferentes versiones. Se trata de un poema de Macías, cuyas variantes - dos de ellas-, se encuentran ya en en los dos testimonios (SA7 y PN1) en los que se recoge integralmente la cantiga del trovador gallego:

\footnotetext{
16 MN8 introduce el fragmento, objeto de ejemplo, en otra posición con respecto a los demás testimonios.
} 


\begin{tabular}{|l|l|l|}
\hline \multicolumn{1}{|c|}{$\mathbf{1}^{\mathbf{a}}$} & $\begin{array}{l}\text { Testimonio } \\
\text { Voema } \\
\text { Macías: SA7 }\end{array}$ & $\begin{array}{l}\text { Con tan alto poderio/ amor nunca fue iuntado/ nin con } \\
\text { tanto ergullo et brio/ como vi por mi pecado }\end{array}$ \\
\hline & $\begin{array}{l}\text { Testimonio } \\
\text { "Querella de } \\
\text { amor" en que } \\
\text { se cita el } \\
\text { poema de } \\
\text { Macías: } \\
\text { MN54 }\end{array}$ & $\begin{array}{l}\text { Con tan alto poderio/ amor nunca fue iuntado/ nin con tant } \\
\text { orgullo brio/ como ui por mi peccado }\end{array}$ \\
\hline
\end{tabular}

\begin{tabular}{|l|l|l|}
\hline \multicolumn{1}{|c|}{$\mathbf{2}^{\mathbf{a}}$} & $\begin{array}{l}\text { Testimonio } \\
\text { poema } \\
\text { MARIANTE } \\
\text { Macías: PN1 }\end{array}$ & $\begin{array}{l}\text { Con tan alto poderyo/ amor nunca fue iuntado/ nin con tal } \\
\text { orgullo et obrio/ qual yo vy por mi pecado }\end{array}$ \\
\hline $\begin{array}{l}\text { Testimonio } \\
\text { "Querella de } \\
\text { amor" en que } \\
\text { se cita el } \\
\text { poema de } \\
\text { Macías: SA7 }\end{array}$ & $\begin{array}{l}\text { Con tan alto poderio/ amor nunca fue iuntado/ ni con tal } \\
\text { ergullo et brio/ Qua yo vi por mi pecado }\end{array}$ \\
\hline
\end{tabular}

Se evidencia de esa manera que este fragmento lírico circulaba en variantes con anterioridad con respecto a la escritura de la "Querella de amor". Puede que el Marqués, a la hora de insertarlo en su decir, eligiera una de estas, bien porque era la versión que tenía delante, bien porque era la versión que en aquel momento recordaba. Merece la pena subrayar que la versión que lee "commo vy yo mal fadado" (versión cuatro, en el primer ejemplo, página 39), no procede de los testimonios que tenemos del poema de Macías; sobre este tema se volverá más adelante. Siguiendo la hipótesis de las variantes de autor, puede que el mismo Santillana en cierto momento decidiera sustituir la cita introducida por la otra versión; de este modo, los testimonios de la "Querella de amor" reflejan exactamente las dos variantes que tenía el texto original de Macías. Habría que considerar también la posibilidad de que la introducción de estas variantes se deba a los mismos copistas, bien porque puede que ellos mismos manejaran las variantes escritas del poema de Macías, bien porque puede que este circulara también oralmente y la memoria del copista le ofreciera una versión diferente de la que le presentaba el texto de Santillana sobre el que trabajaba.

Se dan casos también en que los fragmentos utilizados en la "Querella de amor", si considerados dentro de su poema originario, nos han llegado en una única versión mientras que son los testimonios del decir del Marqués los que registran diferentes variantes. Es este el caso de unos versos de Pero González de Mendoza, 
abuelo de Santillana, recogidos en dos cancionero diferentes (MN15 y PN1) que, a parte de algunas variantes gráficas, reproducen exactamente el mismo texto:

\begin{tabular}{|l|l|}
\hline Testimonio poema P. G. Mendoza: MN15 & Testimonio poema P. G. Mendoza: PN1 \\
\hline Pero te siruo sin arte & Pero te syruo syn arte \\
\hline ay amor amor amor & ay amor amor amor \\
\hline grand cuyta de mi parte & grant cuyta de mi parte \\
\hline
\end{tabular}

Mientras que los únicos testimonios del decir del Marqués que recogen la cita leen lo siguiente:

\begin{tabular}{|l|l|l|}
\hline Versión 1, MN8 & Versión 2, SA7 & Versión 3, ME1 \\
\hline Pero te siruo sin arte & Pero te siruo sin arte & Pero te siruo sin arte \\
\hline ay amor amor amor & ay amor amor & ay amor amor asi \\
\hline $\begin{array}{l}\text { gran cuyta de mi nunca se } \\
\text { parte }\end{array}$ & $\begin{array}{l}\text { gran cuyta de mi nunca se } \\
\text { parte }\end{array}$ & grande cuita de mi \\
\hline & & nunca jamas se parte \\
\hline
\end{tabular}

Las posibilidades en este caso son amplísimas. Por un lado, puede que el mismo Marqués decidiera intervenir sobre el fragmento modificándolo por razones estilísticas o de metro. A este propósito resulta muy interesante cuanto expone Isabella Tomasetti que, al hablar de los decires con cita, subraya la dificultad de identificar los textos intercalados, y de posibles intervenciones sobre estas citas "hechas en el momento mismo de la inserción, es decir de manera totalmente contingente, a veces motivada por la dificultad de la integración." ${ }^{17}$ Por el otro lado, quizás se trate de variantes de autor, del autor originario, es decir, el abuelo del Marqués, que Santillana tenía delante pero que hoy en día no nos han llegado. Puede también que el copista (o los copistas) pudiera manejar estas variantes de Pero González e introducir a su antojo en el texto la que mejor le pareciera y, una vez más, hay que considerar también el factor de la oralidad, ya que bien el Marqués, bien un copista, podrían haber retenido en su memoria una versión quizás incorrecta con respecto a la original de Pero González, introduciéndola después en el texto de la "Querella de amor". También podría pensarse que se trata de un error en la propia transmisión del poema del Marqués, aunque me parece esta última la menos posible de las hipótesis.

Como se puede notar, la complejidad de los problemas que plantea este decir narrativo son numerosos y mi propósito, lejos de buscar una solución a ellos, ha

17 I. Tomassetti (2000), p. 1727. 
sido meramente el de presentarlos y proponer posibles caminos a seguir, ya que, a pesar de que el Marqués de Santillana sea una de las figuras más estudiadas en el panorama literario español, no ha agotado, a mi parecer, la posibilidad de seguir siendo fuente de nuevos hallazgos y aclaraciones.

En última instancia considero importante mencionar un problema añadido alrededor de la transmisión de este poema: la muy poca consideración y relieve que se ha atribuido a ML3 cuya existencia parece haber sido siempre relegada en un segundo plano, probablemente por ser un descriptus, o por ser copia tardía o por representar solo parcialmente la obra del Marqués.

Pérez Priego relaciona indirectamente ML3 con SA8 y MN8. Estos últimos dos códices, como vimos anteriormente, se hacen derivar de un cancionero de autor. Sabemos que SA8 es copia de este cancionero de autor y que MN8, copia tardía del siglo XVI, también tuvo que descender de él y que, a su vez, no basó su copia en $\mathrm{SA} 8^{18}$. Se considera del mismo modo que ML3 pueda derivar, aunque solo en parte, de este cancionero de autor que hoy en día no poseemos, aunque el mismo Pérez Priego afirma que, en el caso de alguno poemas, "ML3 es copia de un testimonio tan tardío como $11 \mathrm{CG}$ "19.

La versión de la "Querella de amor" que nos transmite ML3, si seguimos estrictamente el orden de las estrofas y de los fragmentos líricos intercalados, hay que incorporarla a la rama de SA8 y MN8. Además, los tres testimonios reproducen los cuatro versos finales de finida. Claramente, un cotejo de los tres testimonios se hace difícil dada la laguna de 47 versos que caracteriza SA8, pero es este mi propósito para poder comprobar, si fuera posible, la relación entre los tres.

En el verso 58, SA8 y ML3 leen contra MN8:

v. 58 Ay amor amor SA8 ML3: ay amor amor amor MN8

Cabe preguntarse si podría considerarse este un error conjuntivo aunque, debido a su tipología, bastante frágil, ya que el copista de ML3 da prueba, en varias ocasiones, de no caracterizarse por el esmero y la atención en la copia del poema en cuestión. De todos modos, si aceptamos esta idea, pese a que el ejemplo citado sea el único error conjuntivo que se da entre ML3 y SA8, los dos testimonios formarían una familia frente a MN8. En este caso, o bien ML3 es copia de SA8, o bien los dos proceden de un subarquetipo que los singulariza frente a MN8. Entre estas dos hipótesis, me inclino por la primera, ya que ML3 presenta errores evidentes frente a SA8, por ejemplo en el primer verso SA8 y MN8 leen contra ML3 que, claramente, se equivoca:

v. 1 Ya la grand/gran noche passaua $S A 8 M N 8$ : Ya la gran noche pasada $M L 3$

Mientras al v. 83:

18 Remito a M. A. Pérez Priego (2008), p. 32 para un explicación de las motivaciones que impiden creer que MN8 sea copia de SA8.

19 Ibíd. 
v. 83 meu ben perder cuytado $S A 8 M N 8$ : meu ben querer perder cuytado $M L 3$

El copista de ML3 introduce el verbo querer, produciendo un verso hipermétrico, probablemente influido por el verso con que el que éste rima: "a meu querer de grado."

Por su parte SA8, no presenta errores separativos tales que impidan, aunque sea sólo una mera conjetura, que haya podido servir como fuente para la copia de ML3.

A su vez, MN8 no presenta errores conjuntivos con respecto a los otros dos testimonios, sino que se caracteriza meramente por un error singular que sugiere situarlo en una rama distinta con respecto a ML3 y SA8. En:

v. 2 E la luna sescondia/se escondia $S A 8 M L 3$ : e la luna sestendia $M N 8$

A la luz de los ejemplos arriba citados resulta difícil, para no decir imposible, poder afirmar algo con absoluta certeza. Ya hemos visto que MN8 y SA8 derivan de la misma copia de autor, aunque en el caso de MN8 se tenga que interponer otra/s copia/s. Un solo error conjuntivo quizás no sea suficiente para poder afirmar con certeza que ML3 deriva de SA8 y, además, tenemos el problema de la laguna de 47 versos que impide una valoración adecuada del tema. ML3 no presenta tal laguna, pero esto, a mi parecer, no es suficiente para poder descartar totalmente la posibilidad de que ML3 pueda derivar directa o indirectamente de SA8, ya que la pérdida del folio puede haberse producido en época posterior a la copia de ML3. Las relaciones entre este último y MN8 tampoco resultan del todo claras, ya que la falta de estudios sobre los dos impide poder ir más allá de meras y aleatorias suposiciones.

\section{Obras citdas}

BLECUA, Alberto: Manual de crítica textual, Madrid, Editorial Castalia, 1983.

Cancionero del Marqués de Santillana [B:U:S:, Ms 2655], Presentación de Pedro M. Cátedra, transcripción de Javier Coca Senande, Salamanca, Universidad de Salamanca, Iberduero, 1990.

El Cancionero de Toledo del Marqués de Santillana, ed. José Luis Pérez López, Toledo, Caja de Toledo, Obra cultural, 1989.

DUTTON, Brian: El cancionero del siglo XV, Salamanca, Universidad de Salamanca, 1990-1991, $7 \mathrm{v}$.

LAPESA, Rafael: La obra literaria del Marqués de Santillana, Madrid, Ínsula, 1956.

SANTILlanA, Marqués de: Obras completa, ed. introd. y notas de Ángel Gómez Moreno y Maximilian P.A.M. Kerkhof, Barcelona, Planeta, 1988.

Santillana, Marqués de: Poesías Completas, ed., estudio y notas de Miguel Ángel Pérez Priego, Madrid, Alhambra, 1983.

Santillana, Marqués de: Poesía lírica, ed. Miguel Ángel Pérez Priego, Madrid, Cátedra, 2008. 
TOMASSETTI, Isabella: "Sobre la tradición ibérica de los decires con citas: apuntes para un estudio tipológico", en Actas del VIII Congreso Internacional de la Asociación Hispánica de Literatura Medieval, Santander, 22-26 de Septiembre de 1999, al cuidado de Margarita Freixas y Silvia Iriso, con la colaboración de Laura Fernández, Santander, Consejería de Cultura del Gobierno de Cantabria, Año Jubilar Labaniego, Asociación Hispánica de Literatura Medieval, 2000.

VARVARO, Alberto: Premesse ad un'edizione critica delle poesie minori di Juan de Mena, Napoli, Liguori, 1964.

MENÉNDEZ PIDAL, Ramón: "Poesías inéditas del Marqués de Santillana", en Poesía árabe y poesía europea, Madrid, Espasa-Calpe, 1941. 